\title{
Profit Maximization and Optimal Bidding Strategies of Gencos in Electicity Markets using Self Adaptive Differential Evolution
}

\author{
B. Rampriya \\ Department of Electrical and Computer Engineering, College of Technology, \\ Debre Markos University, Debre Markos, Ethiopia.
}

\begin{abstract}
This paper presents the solution for the suppliers' aims to achieve profit more than the rivals participating in the competition. The supplier (decision maker) optimization problem is formulated and their bid quantities are optimized using Self adaptive Differential Evolution $(\mathrm{SaDE})$. A six unit system is used to illustrate the methodology for a single trading period with both elastic and inelastic loads. The performance of the test systems under perfect and oligopoly market situations are compared to show the importance of optimization of bidding parameters in deregulated markets. Numerical results illustrate the effectiveness of the method in solving the supplier profit maximization problem.
\end{abstract}

Keywords: Differential Evolution (DE), Generation Companies (GENCOs), Market Clearing Price (MCP), System Operator (ISO), Power eXchange (PX).

\section{Introduction}

The deregulation of the power industry and setting up of open markets for electricity has led to a separation between generation, transmission and distribution activities. The basic aim of the deregulation is to create competition among generating units (GENCOs) to deliver reliable power supply to consumers. Deregulation results in a competitive structure among generation companies either through auction markets or through bilateral/multi-lateral mechanisms. The GENCOs in deregulated system challenges to produce electric power with an objective of maximizing their individual profit. This solution to profit maximization problem has been presented in various research papers. GA is used to achieve maximum profit of GENCOs participating in competitive environment [1]. Profit based unit commitment problem is solved using hybrid LR-EP and the amount of power to be sold in power and reserve markets are also presented [2]. These methods proposed the profit maximization of GENCOs without the consideration of bids submitted by the suppliers.

The supplier profit maximization problem is done by determining the bidding parameters of the market participants. Most of the researchers have determined the bidding parameters by Monte Carlo and probability density to determine the rival's bidding parameters [3]. These probabilistic methods require large amounts of observations which are the available historical data for our problem. The problem of building optimally coordinated bidding strategies for competitive suppliers in day-ahead energy and spinning reserve is addressed in [4]. In this paper, Refined Genetic Algorithm (RGA) is used to build the optimally coordinated bidding strategies. The bidding parameters are optimized using stochastic optimization Evolutionary Programming (EP) technique and are discussed in [5].

Rather than crisp set, the bidding parameters are treated as fuzzy sets and presented only preliminary works and a systematic method is not developed in [6]. A possibility theory based approach is presented to build the optimal bidding strategies in a single auction trade period and inter-temporal constraint such as start-up and shut down of a generator and multi hour trade task with up-time and down time constraints of generators are not included in [7].

In this paper, the bidding decision problem for the decision maker (supplier) is formulated. Before submitting bids to pool operators, the supplier optimizes their bidding data with an objective of profit maximization and then the optimized bids are submitted to the ISO. In the sealed bid auction, the data of rivals' value are confidential. The supplier participating in competitive markets tries to estimate the rivals' value but it is difficult to predict the same.

Received: May $6^{\text {th }}, 2015$. Accepted: December $13^{\text {rd }}, 2016$ 


\section{B. Rampriya}

However the past data are available. The suppliers are also able to calculate the rival's bidding value by using joint or multivariate Probability Distribution Function (PDF). These situations and also the solution for optimal bidding of the suppliers are explained in this paper. The optimal values of supplier bid quantities are obtained and the supplier profit is determined using Self-adaptive Differential Evolution (SaDE). The optimal values of supplier bid quantities are obtained to maximize its own profit was determined using SaDE.

Even though the most suitable strategy and the control parameters are identified and applied to a problem using Differential Evolution (DE) technique, it may require a huge amount of computation time. Different strategies and corresponding parameter settings with different global and local search capability might also be preferred during different evolution stages [8].The Self-adaptive Differential Evolution (SaDE) algorithm can automatically adapt the learning strategies settings during evolution.

This paper is organized as follows: Section 2 presents the market clearing mechanism in competitive energy markets, section 3 problem statement of profit maximization of decision makers submitting bids to market operators, section 4 deals with the solution methodology to find the optimum schedules of the supplier, section 5 presents the results and discussions, and section 6 concludes.

\section{Market Clearing Mechanism in A Competitive Environment}

The market structure considered in this paper is single sided auction mechanism in which the suppliers are alone allowed to participate in the energy markets. They can submit their supply bids to energy bids to spot energy markets.

\section{Energy or spot markets:}

The suppliers participating in electricity markets is required to bid a linear non-decreasing energy function to ISO. Upon receiving bids from the suppliers, the pool determines a set of generation outputs that meets the load demand and minimizes the total cost spent by bidder.

The i-th supplier energy bidding function can be represented as

$$
\begin{aligned}
& B_{i}^{(t)}\left(P_{i t}\right)=\alpha_{i}^{(t)}+\beta_{i}^{(t)} P_{i t} \quad \mathrm{t}=1,2, \ldots \mathrm{T} \\
& \sum_{i=1}^{N} P_{i}^{(t)}=D_{t} t=1,2, \ldots T \\
& P_{i \min } \leq P_{i t} \leq P_{i \max }
\end{aligned}
$$

where $\mathrm{D}_{\mathrm{t}}$ is the demand at hour $\mathrm{t}, \alpha_{i}^{(t)}, \beta_{i}^{(t)}$ are the intercept and slope of the energy bidding curve of the suppliers respectively, $\boldsymbol{P}_{i t}$ is the power generation output within the set of capacity limits $\boldsymbol{P}_{\boldsymbol{i} \text { min and }} \boldsymbol{P}_{\boldsymbol{i} \text { max }}$.

The Market Clearing Price (MCP) for power is calculated $\mathrm{t}=1,2, \ldots \mathrm{T}$

The power dispatch by each supplier can be calculated as,

$$
P_{i t}=\left(M C P_{t}-\alpha_{i}^{(t)}\right) / \beta_{i}^{(t)} \quad \mathrm{i}=1,2 \ldots \mathrm{N}
$$

Where $\mathrm{N}$ is the total number of suppliers participating in energy markets. The market is cleared and the price is quoted by ISO. The power dispatched by each supplier is checked for their maximum and minimum capacity limits. If the supplier is not able to provide minimum 
power requirement, then the corresponding supplier is not allowed to participate in the competition i.e., If $\mathrm{P}_{\mathrm{it}}<\mathrm{P}_{\mathrm{imin}}$ then $\mathrm{X}_{\mathrm{it}}=0$ else $\mathrm{X}_{\mathrm{it}}=1$. Thus unit commitment decisions are well taken into account. If the supplier violates its maximum limit, the maximum power is fixed and it is no longer be able to a marginal generator.

\section{Supplier Profit Maximization Problem}

The profit maximization objective of suppliers participating in energy markets and competing with the other suppliers can be stated as,

Maximize: Profit, $\mathrm{PF}=$ Revenue, RV- Total cost, TC

$$
P F=\sum_{i=1}^{N} \sum_{t=1}^{T}\left[M C P_{t} \cdot P_{i t}-C_{i}\left(P_{i t}\right)\right] X_{i t}
$$

The ON/OFF status of the suppliers decided by ISO and $C^{i}\left(\mathrm{P}^{\mathrm{it}}\right)$ is the fuel cost function of the suppliers. The constraints included are a) Power balance and b) Minimum and maximum capacity limit of the suppliers.

a) Power balance constraints

The power balance constraint is an equality constraint that reduces the power system to a basic principle of equilibrium, between total generation of GENCO participating in the electricity markets and demand profile of the customers.

$$
\sum_{j=1}^{N}\left(P_{i t}\right) X_{i t}=\sum_{t=1}^{T} D_{t} t=1,2, . . T
$$

b) Supply limit constraints

Generation units have lower and upper production limits that are directly related to the generator design. These bounds can be defined as a pair of inequality constraints $P_{i \min } \leq P_{i t} \leq P_{i \max }$

\section{Solution Methodology}

The solution methodology used in the present study for solving the optimal bidding problem is explained as mentioned below.

1) Initialization

The SaDE counter is initialized and maximum number of iteration is specified.

2) Creation of parent population

The initial population of the bidding coefficient of the supplier to be optimized is generated within the search limits.

3) Calculation of bidding coefficients of rivals'

The rivals' bidding coefficients are determined using multivariate PDF.

4) Calculation of MCP

The MCP is calculated with the bidding data of suppliers and rivals' using equation (4).

Based on the market price, $\mathrm{P}$ it from equation (5) is calculated and limit values are checked.

5) Determination of unit ON/OFF status

If $\mathrm{P}_{\mathrm{it}}<\mathrm{P}_{\mathrm{imin}}$ then $\mathrm{X}_{\mathrm{it}}=0$ else $\mathrm{X}_{\mathrm{it}}=1$. Thus the unit ON/OFF $\mathrm{X}_{\mathrm{it}}$ status can be calculated by taking an account of the constraints to be satisfied in all trading periods.

6) Economic dispatch

With the calculated $X_{i t}$, the optimal dispatch of power $P_{i t}$ is calculated using Quadratic

Programming $(\mathrm{QP})$. The revenue generated and fuel costs spent are determined.

7) Calculation of fitness and $\mathrm{SaDE}$ operations

The fitness is calculated as per equation (6) and the mutation, crossover and selection 
operations are performed. Mutation process is done by selecting the best strategy among the five strategies as per the highest learning probability in all iterations. Thus SaDE is incorporated to improve the solution quality and the psudo code of $\mathrm{SaDE}$ is given in Appendix as Table 1

8) Stopping criteria

The steps from 1 to 6 are repeated until the specified maximum number of iterations is reached.

\section{Results and Discussion}

To illustrate the perfect competition and optimal bidding strategy, a test system with six suppliers are taken. The market situations are analyzed for test system participating in single hour trading. The results of test system under perfect competition and optimal bidding strategy are tabulated. The generator data for Test system-1 is taken from [9] and given in Appendix as Table A-3. The best values of SaDE parameters are chosen by the experiments carried out with different values of parameters and are set as $\mathrm{CR}=0.9, \mathrm{~F}=0.5, \mathrm{NP}=200$, MAXITER=200.

The test system with six generators is used to illustrate the solution methodology of profit maximization under perfect competition and oligopoly markets (optimal bidding strategies). Consider a situation in which the supplier-1 aims to maximize its own profit when compared to other suppliers-2, 3, 4 and 5 (rivals') in the market. This example system is utilized for single hour load demand of $390 \mathrm{MW}$. The fuel cost equation is expressed in quadratic form [10] as,

$$
C_{i}\left(P_{i t}\right)=\frac{1}{2} a_{i}\left(P_{i t}\right)^{2}+b_{i} P_{i i}
$$

\section{Case A: Perfect competition}

In a perfect competitive market without optimal bidding, the bidding parameters $\beta^{\mathrm{i}}$ and $\alpha^{\mathrm{i}}$ of suppliers are assumed to be equal to that of the cost coefficients $a^{i}$ and $b^{i}$ respectively [11]. With the bidding data, MCP is calculated using eq. (4). At the market equilibrium, power dispatch and UC (pool scheduling) is done by ISO as mentioned in step 4 and 5 of Section 4. With the unit status, the constraints are checked and the economic dispatch of all the suppliers with an objective of maximizing the profit of supplier-1 is determined using QP. The results for this test system under perfect competition market situation are shown in Table 1.

\section{Case B: Oligopoly- Optimal bidding strategy}

In an oligopoly market, some suppliers act as price makers and the others as price takers [12]. In this condition, each supplier will try to maximize its own profit. For a supplier, it is critical to devise a good bidding strategy according to its opponents' bidding behavior, the model of demand, market mechanism, and power system operating conditions. To achieve maximum profit, the supplier-1 decides to optimize its bidding parameters using an optimization algorithm. It is assumed that the supplier-1 bids the true value of the slope, $\beta^{1}$ and it bids in a linear, non-decreasing supply bid curve that varies the intercept $\alpha^{1}$ [13]. Therefore the bidding parameter of supplier- $1 \beta^{1}$ is fixed as $a^{1}$ which is optimized within the intervals $b^{1}$ and $\mathrm{m} \cdot \mathrm{b}^{1}$ ( $\mathrm{m}$ is set to be 2 in all simulations) using SaDE algorithm. The bidding parameters of rivals' ( $\beta^{\mathrm{i}}$ and $\alpha^{\mathrm{i}}$ where $\mathrm{i}=2,3,4,5$ and 6 ) are generated according to joint or multivariate normal distribution as explained in Appendix as Table A-2. With the optimized bid of supplier1 and multivariate distributed rivals' bids, MCP, power dispatch and corresponding unit status are calculated. With the unit status, the constraints specified are checked and economic power dispatch is found for a load demand of $390 \mathrm{MW}$ using QP. 
Table 1. Results of 6 unit single hour data under perfect competition and oligopoly markets

\begin{tabular}{|c|c|c|c|c|c|c|c|c|}
\hline \multirow{2}{*}{$\begin{array}{c}\text { Market } \\
\text { situations }\end{array}$} & \multirow{2}{*}{$\begin{array}{c}\mathrm{MCP} \\
(\$)\end{array}$} & \multirow{2}{*}{ Outputs } & \multicolumn{6}{|c|}{ Suppliers } \\
\hline & & & 1 & 2 & 3 & 4 & 5 & 6 \\
\hline \multirow{6}{*}{$\begin{array}{c}\text { Perfect } \\
\text { competition }\end{array}$} & \multirow{6}{*}{3.427} & Power dispatch (MW) & 160 & 48.11 & 38.94 & 28.94 & 85.06 & 28.94 \\
\hline & & $\alpha$ & 2 & 1.75 & 1 & 3 & 3.15 & 3 \\
\hline & & $\beta$ & 0.0087 & 0.035 & 0.0625 & 0.015 & 0.003 & 0.015 \\
\hline & & Fuel cost $(\$)$ & 432 & 124.7 & 86.344 & 93.100 & 280.0 & 93.10 \\
\hline & & Revenue (\$) & 548.40 & 164.9 & 133.49 & 99.191 & 291.5 & 99.19 \\
\hline & & Profit (\$) & 116.40 & 40.11 & 47.14 & 6.09 & 11.52 & 6.09 \\
\hline \multirow{6}{*}{$\begin{array}{c}\text { Oligopoly } \\
\text { (Optimal } \\
\text { bidding } \\
\text { strategy) }\end{array}$} & \multirow{6}{*}{3.912} & Power dispatch (MW) & 160 & 48.11 & 38.94 & 28.94 & 85.06 & 28.94 \\
\hline & & $\alpha$ & 3.04 & 2.74 & 1.41 & 0.57 & 1.01 & 2.64 \\
\hline & & $\beta$ & 0.0087 & 0.02 & 0.021 & 0.07 & 0.053 & 0.07 \\
\hline & & Fuel cost (\$) & 432 & 124.7 & 86.34 & 93.10 & 280.0 & 93.10 \\
\hline & & Revenue (\$) & 625.8 & 188.2 & 152.3 & 113.2 & 332.7 & 113.2 \\
\hline & & Profit (\$) & 193.8 & 63.5 & 65.99 & 20.10 & 52.70 & 20.1 \\
\hline
\end{tabular}

The revenue, cost and profit calculations are done and the results of this case are given in Table 1. Figure 1 shows the comparison of supplier profit under both the cases A and B. It is observed that the profit of supplier-1 under perfect competition and oligopoly may come to $\$ 116.401$ and $\$ 193.87$ respectively. Thus there is a rise in profit of $\$ 77.469$ if the supplier incorporates an optimal bidding strategy before submitting bids to ISO. This is due to the additional revenue generated by the suppliers by an increase in MCP from $\$ 3.427$ to $\$ 3.912$.

Table 2. Comparison of results of perfect competition and optimal bidding strategy

\begin{tabular}{|c|c|c|c|}
\hline \multicolumn{2}{|c|}{ Perfect competition } & \multicolumn{2}{c|}{ Oligopoly (Optimal bidding strategy) } \\
\hline Profit of supplier-1(\$) & $\begin{array}{c}\text { Execution } \\
\text { time (sec) }\end{array}$ & Profit of supplier-1 (\$) & $\begin{array}{c}\text { Execution } \\
\text { time (sec) }\end{array}$ \\
\hline 108(Rajathy et al. 2010) & -- & 192(Rajathy et al. 2010) & -- \\
\hline 116.40 & 0.2 & 193.87 & 15.46 \\
\hline
\end{tabular}

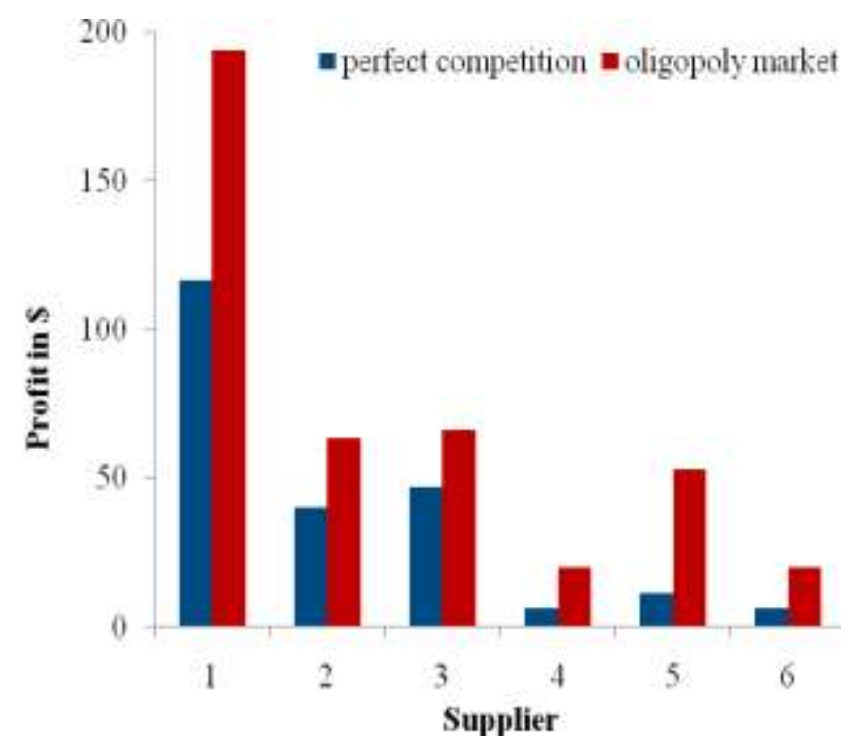

Figure 1. Comparison of profits for six suppliers for case A and B 


\section{B. Rampriya}

Table 2 shows the comparative results of the methods used to determine the profit of supplier-1 under perfect competition and oligopoly markets with an execution time of 0.2 and 15.46 seconds respectively. In case of oligopoly markets, the rise in execution time is due to repeated simulation up to 200 iterations to optimize the bids until the objective of maximum profit is achieved. The bid coefficients are optimized using SaDE and the supplier-1 can get a profit of $\$ 193.87$. Since the mutation process has been improved by introducing learning strategies, the profit obtained is $\$ 193.87$ more than traditional DE, which was $\$ 192$. The idea behind our proposed learning strategy adaptation is to probabilistically select one out of several available learning strategies and apply to the current population.

\section{Conclusion and Future Expansion}

$\mathrm{SaDE}$ algorithm with learning strategies to build optimal bidding strategy for power suppliers participation for single and multi-hour trading in single auction pool-co markets is proposed. This algorithm can automatically adapt its learning strategies and the associated parameters during the evolving procedure. The power dispatch level and UC of suppliers participating in energy markets are determined. The optimized values of bids of the suppliers are found in such a way that the suppliers do yield profit and get benefited. The rivals' bidding behavior has been determined from multivariate probability distribution function. Simulation results prove the capability of $\mathrm{SaDE}$ to solve this supplier optimization problem effectively. The method developed in this research provide a systematic way of investigating the profit maximization and bidding strategies of the suppliers participating in energy markets. This method can be extended for the market participants that are able to provide the required ancillary services by setting up reserve markets.

\section{Acknowledgement}

The author is grateful to acknowledge the support from the Department of Electrical Engineering, College of Technology, Debre Markos University, Debre Markos, Ethiopia.

\section{APPENDIX}

The pseudo code of self adaptive differential algorithm, joint or multi variate probability distribution function, generator data of six unit test system are given in Tables 1, 2 and 3.

Table 1. Pseudo code of Self adaptive differential algorithm

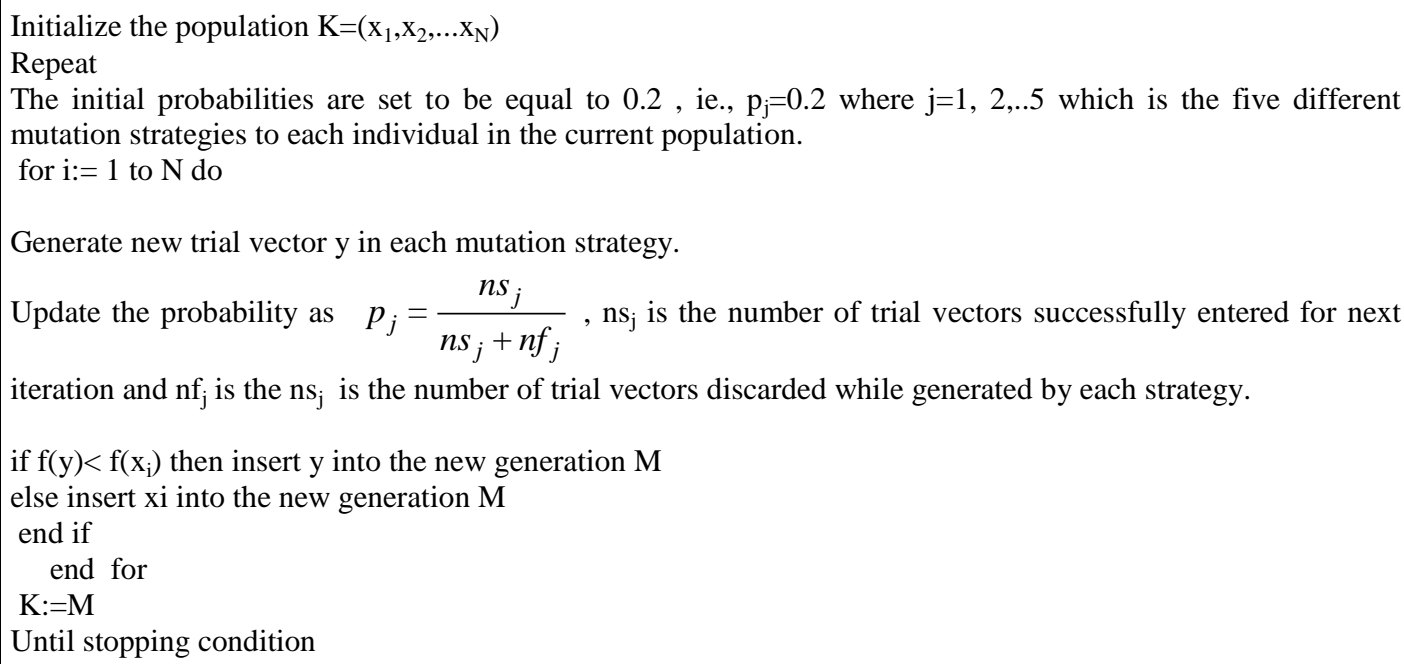


Table 2. Joint or multivariate probability density function

The basics of joint PDF are given below

Let $\mathrm{x}$ and $\mathrm{y}$ be distributed bivariate normal. $\mathrm{x}$ and $\mathrm{y}$ are said to be distributed bivariate normal if they are linear functions of other two independent normal random variables [14].

The joint probability distribution function is given by $\mathrm{f}(\mathrm{x}, \mathrm{y})=$

$$
\frac{1}{2 \pi \sigma_{X} \sigma_{Y} \sqrt{1-\sigma^{2}}} \exp \left\{-\frac{1}{2\left(1-\rho^{2}\right)}\left[\frac{\left(x-\mu_{X}\right)^{2}}{\sigma_{X}}+\frac{\left(y-\mu_{Y}\right)^{2}}{\sigma_{Y}}-\frac{2 \rho\left(x-\mu_{X}\right)\left(y-\mu_{Y}\right)}{\sigma_{X} \sigma_{Y}}\right]\right\}
$$

where $\mathrm{E}[\mathrm{x}]=\mu_{\mathrm{x}}, \mathrm{E}[\mathrm{y}]=\mu_{\mathrm{y}}, \mathrm{SD}(\mathrm{x})=\sigma_{\mathrm{x}}, \mathrm{SD}(\mathrm{y})=\sigma_{\mathrm{Y}}, \rho=\operatorname{cor}(\mathrm{x}, \mathrm{y})$ and the variables $\mathrm{x}$ and $\mathrm{y}$ are expressed in compressed form as

$$
(x, y) \sim N\left\{\left[\begin{array}{l}
\mu_{X} \\
\mu_{Y}
\end{array}\right],\left[\begin{array}{ll}
\left(\sigma_{X}\right)^{2} & \rho \sigma_{X} \sigma_{Y} \\
\rho \sigma_{X} \sigma_{Y} & \left(\sigma_{Y}\right)^{2}
\end{array}\right]\right\}
$$

This joint PDF is utilized to estimate the rivals' bidding coefficients as mentioned below.

The bid coefficients of rivals' participating in energy markets, $\beta_{\mathrm{i}}$ and $\alpha_{\mathrm{i}}(\mathrm{i}=1,2 \ldots \mathrm{N})$ obey a multivariate normal distribution with the PDF given in (Fushuan Wen and A. Kumar David 2001) and can be expressed in compressed form as

$$
\left(\alpha_{i}^{(t)}, \beta_{i}^{(t)}\right) \sim N\left\{\left[\begin{array}{l}
\mu_{i, t}^{(\alpha)} \\
\mu_{i, t}^{(\beta)}
\end{array}\right],\left[\begin{array}{ll}
\left(\sigma_{i, t}^{(\alpha)}\right)^{2} & \rho_{i, t} \sigma_{i, t}^{(\alpha)} \sigma_{i, t}^{(\beta)} \\
\rho_{i, t} \sigma_{i, t}^{(\alpha)} \sigma_{i, t}^{(\beta)} & \left(\sigma_{i, t}^{(\beta)}\right)^{2}
\end{array}\right]\right\}
$$

where $\rho_{j, t}$ is the correlation coefficient between $\alpha_{i}^{(t)}$ and $\beta_{i}^{(t)}, \mu_{i, t}^{(\alpha)}, \mu_{i, t}^{(\beta)}, \sigma_{i, t}^{(\alpha)}$ and $\sigma_{i, t}^{(\beta)}$ are the parameters of the multivariate normal distribution. The supplier who is aware of market power in deregulated market is likely to bid above the marginal production cost. The rivals' are expected to bid $20 \%$ above operating cost. The mean, standard deviation and correlation factor of rivals' in energy markets are estimated as

$$
\begin{aligned}
& \mu_{i, t}^{(\alpha \alpha}=1.2 b_{i}, \mu_{i, t}^{(\beta \beta}=1.2 \times 2 a_{i} \\
& 4 \sigma_{i, t}^{\alpha \alpha \alpha}=0.15 b_{i}, 4 \sigma_{i, t}^{(\beta \beta}=0.15 a_{i} \\
& \rho_{i, t}=-0.1
\end{aligned}
$$

The mean and standard deviation of $\alpha_{i}$ and $\beta_{i}$ are specified as $\left.\mid \mu_{i, t}^{(\alpha \alpha}-4 \sigma_{i, t}^{(\alpha \alpha}, \mu_{i, t}^{(\alpha \alpha}+4 \sigma_{i, t}^{(\alpha \alpha}\right\rfloor$ and $\left\lfloor\mu_{i, t}^{(\beta \beta}-4 \sigma_{i, t}^{(\beta \beta}, \mu_{i, t}^{(\beta \beta}+4 \sigma_{i, t}^{(\beta \beta}\right\rfloor$ respectively, with the probability of 0.999 . The rivals' bid data and their corresponding joint normal distribution functions are estimated using 'mvnrnd' and 'mvnpdf' commands respectively in MATLAB and then given as the input to the SaDE algorithm. 
Table 3. Generator data of six unit system

\begin{tabular}{|c|c|c|c|c|c|}
\hline Gen No. & $\begin{array}{c}a_{p i} \\
\left(\$ / \mathrm{MWh}^{2}\right)\end{array}$ & $\begin{array}{c}b_{p i} \\
(\$ / \mathrm{MWh})\end{array}$ & $\begin{array}{c}C_{p i} \\
(\$)\end{array}$ & $\begin{array}{c}\mathrm{P}_{\min } \\
(\mathrm{MW})\end{array}$ & $\begin{array}{c}\mathrm{P}_{\max } \\
(\mathrm{MW})\end{array}$ \\
\hline 1 & 0.00875 & 2 & 0 & 50 & 160 \\
\hline 2 & 0.035 & 1.75 & 0 & 20 & 100 \\
\hline 3 & 0.0625 & 1 & 0 & 30 & 80 \\
\hline 4 & 0.015 & 3 & 0 & 10 & 60 \\
\hline 5 & 0.00334 & 3.15 & 0 & 50 & 160 \\
\hline 6 & 0.015 & 3 & 0 & 10 & 50 \\
\hline
\end{tabular}

\section{References}

[1]. Charles W.Ritcher, Gerald B.Sheble, A Profit based unit commitment GA for the competitive environment, IEEE Trans. on Power Systems 15 (2) (2000) 715-721.

[2]. Pathom Attaviriyanupap, Hiroyuki kita, Jun Hasegawa, "A Hybrid LR-EP for solving new profit based UC problem under competitive environment", IEEE Transactions on Power Systems 18 (1) (2003)229-236, 2003.

[3]. Wen.F, A. K. David, "Optimal bidding strategies and modeling of imperfect information among competitive generators", IEEE Trans. on power systems, Vol. 16, No.1, pp. 15-21, 2001.

[4]. Fushuan Wen, A.K.David, "Coordination of bidding strategies in day-ahead energy and spinning reserve markets", Int. J. of Electric Power and Energy systems, 24 (2002), 251261.

[5]. PathomAttaviriyanupap, Hiroyuki Kita, Eiichi Tanaka and Jun Hasegawa, "New bidding strategy formulation for day ahead energy and reserve markets based on evolutionary programming", International Journal of Electrical power and energy systems, Vol. 27, pp. 157-167, 2005.

[6]. C. Mattson, D.Lucarella, and C.C. Liu, "Modelling a competitor's bidding behavior using fuzzy interface networks", in Proc of International conference on Intelligent systems Applications in Power systems (ISAP), 2001.

[7]. Li Yung, Fushuan Wen, F.F. Wu, Yixin Ni and Jiaju Qiu, “ Development of Bidding strategies in Electricity Markets Using Possibility Theory”, pp. 182-187,2002.

[8]. Qin A. K and P. N. Suganthan, "Self-adaptive differential evolution algorithm for numerical optimization," Proceedings of the 2005 IEEE Congress on Evolutionary Computation, vol. 2, pp. 1785-1791, 2005.

[9]. Rajathy R, Gnanadass R, Ramaraaj J, Rajesh R, Kishore U and Harish kumar, (2010), "Bidding strategy using differential evolution in a day-ahead market considering unit commitment problem", Proceedings of International conference on Control, Communication and Power Engineering, pp. 103-110.

[10]. N. Deeb, "Simulated annealing in power systems," in Proceedings of IEEE International Conference of Manand Cybernetics, Vol. 2, pp.1086-1089, October 1992.

[11]. George Gross and David Finlay, "Generation supply bidding in perfectly competitive Electricity Markets", Computational and Mathematical Organization Theory, Vol. 6, pp. 83-98, 2000.

[12]. Soleymani. S, "Bidding strategy of generation companies using PSO combined with SA method in the pay as bid markets", Elect. Power and Energy Syst., Vol. 33, pp. 12721278, 2011.

[13]. Halli song, Chen -Ching Liu, Jacques Lawarree, "Decision making of an electricity supplier's bid in a spot market", in IEEE conference proceedings, pp.692-696, 1999.

[14]. Bertsekas D.P and J. N. Tsitsiklis, (2002), 'Introduction to probability', Athena Scientific Publishers, I edition. 


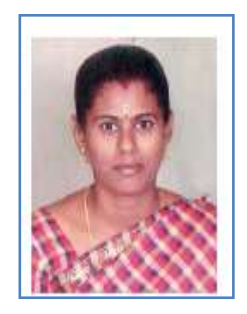

B. Rampriya was born in Tirunelveli, located in southern part of the state of Tamilnadu, India, 1978. She was graduated in Electrical and Electronics Engineering and post graduated in Power Systems Engineering in 2000 and 2004 respectively. She was awarded with $\mathrm{Ph} . \mathrm{D}$ degree from Kalasalingam University in the year 2014. Presently, she is a faculty member in the department of Electrical and Computer Engineering, College of Technology, Debre Markos University, Ethiopia. Her field of interest includes Power System Operation and Control, Power System Optimization and Evolutionary Computation. 